\title{
Um Estudo de Caso de Avaliação de Acessibilidade de Documentos PDF de um Ambiente Virtual de Aprendizagem
}

\author{
Letícia Mariane Morais Silva \\ Departamento de Computação e \\ Sistemas - Universidade Federal de \\ Ouro Preto \\ leticiamms.contato@gmail.com
}

\author{
Lucinéia Souza Maia \\ Departamento de Computação e \\ Sistemas - Universidade Federal de \\ Ouro Preto \\ lucineia@ufop.edu.br
}

\author{
Gilda Aparecida de Assis \\ Departamento de Computação e \\ Sistemas - Universidade Federal de \\ Ouro Preto \\ gildaaa@ufop.edu.br
}

\begin{abstract}
Virtual learning environments are commonly adopted by universities as classroom teaching support. One of the resources they offer are class presentations, usually in PDF files. Considering that web accessibility is essential to allow users with some limitations to understand, interact, operate and navigate web content, this article presents an assessment of the accessibility of PDF documents made available on Moodle. The methodology used a hybrid approach comprised techniques and practices for assessing human-computer interaction and assessing accessibility with users simulating blindness. A quantitative analysis of the data was carried out considering the accessibility problems faced by users in elements such as tables, graphs, mathematical formulas, expressions in foreign languages and presentation topics. The results indicated accessibility problems in all documents in the sample, even causing users to drop out during the evaluations.
\end{abstract}

\section{KEYWORDS}

Acessibilidade Web, acessibilidade de PDF, documentos PDF, avaliação de acessibilidade, avaliação com usuários

\section{INTRODUÇÃO}

A crescente oferta de serviços na Web e em tecnologias mobile, tem feito com que as pessoas utilizem com mais frequência a Internet para a realização de trabalhos, estudos, negócios e entretenimento. Contudo, em algum momento, alguns usuários podem enfrentar problemas de acesso à informação devido às barreiras de acessibilidade Web. Essas barreiras, de acordo com o World Wide Web Consortium (W3C), impedem os usuários Web de perceber, entender, navegar, interagir e contribuir com a Web[1].

Barreiras de acessibilidade Web podem ser verificadas em decorrência de dificuldades particulares a respeito de característica tecnológicas, tal como qualidade da Internet durante o acesso à Web; fatores ambientais, como por exemplo, ambiente com muita luz e ruído e; características dos usuários, como comprometimento físico, sensorial e/ou intelectual ${ }^{1}$. Desse modo, o conteúdo Web deve seguir recomendações para que os usuários consigam realizar suas atividades com êxito.

O conteúdo Web é criado utilizando linguagem de marcação, de estilos e/ou de programação. Esse conteúdo pode ainda ser apresentado ao usuário em documentos como o Portable Document File (PDF). Documentos PDF são compatíveis com diferentes tipos

\footnotetext{
${ }^{1}$ Neste artigo, o termo "pessoa com comprometimento" será utilizado no lugar de "pessoa com deficiência", pois entende-se que a defiência está no ambiente ou meio, e não no indivíduo.
}

de hardware e software. Logo, eles preservam as características originais de apresentação da informação independente do meio de acesso pelo usuário.

Devido a estas características de robustez e portabilidade, o PDF é amplamente utilizado no contexto do ensino superior para o compartilhamento de materiais de apoio aos estudantes por meio, por exemplo, de Ambientes Virtuais de Aprendizagem (AVA). Nesse sentido, é importante que os materiais em PDF disponibilizados nessas plataformas sejam acessíveis, assim como as páginas Web. Logo, o objetivo deste artigo é apresentar uma avaliação de acessibilidade de materiais de apoio disponibilizados em PDF no AVA Moodle por professores de cursos da área de tecnologia de uma universidade.

O artigo está organizado da seguinte forma: a Seção 2 apresenta a metodologia utilizada na realização da pesquisa. A Seção 3 aborda a fundamentação teórica sobre acessibilidade de documentos PDF. A Seção 4 apresenta o estado da arte sobre avaliação de acessibilidade em PDF com as publicações mais recentes sobre o assunto. O estudo de caso realizado é detalhado na Seção 5. Por fim, a seção 6 apresenta as considerações finais.

\section{METODOLOGIA}

O estudo é uma pesquisa exploratória, pois busca identificar objetos de estudo dentro do contexto de acessibilidade de PDF, além da problematização acerca da interação de usuários sem a visão com esse tipo de documento. Trata-se de uma pesquisa de natureza qualitativa, uma vez que esta não se preocupa com representatividade numérica, mas com a compreensão de um grupo social com características específicas.

Quanto aos procedimentos metodológicos, utilizou-se a pesquisa bibliográfica e o estudo de caso. A pesquisa bibliográfica foi realizada em dois momentos: (1) no levantamento bibliográfico da fundamentação teórica-metodológica e; (2) na revisão de literatura para o levantamento de trabalhos publicados sobre avaliação de PDF nos últimos anos.

A revisão de literatura baseou-se na abordagem de Okoli e Schabram [2]. Nesse sentido, determinou-se: (1) as bases de dados bibliográficas da Ciência da Computação e Educação; (2) a temporalidade das publicações de 2015 a 2020 e; (3) as seguintes expressões de busca: "accessibility AND "Portable Document File""; "(web accessibility) AND PDF"; ""accessible PDF"”. Dos resultados da busca, selecionou-se publicações pelos seus títulos, excluindo aqueles que abordavam outros aspectos da acessibilidade de PDF como a criação de PDF acessível. Em seguida, os artigos resultantes foram analisados de acordo com seus resumos, considerando aqueles que eram mais relacionados à proposta deste artigo. Para artigos selecionados nessa fase, realizou-se uma leitura mais apurada para identificar a 
correlação com esta pesquisa. Estes artigos estão descritos na Seção 4.

O estudo de caso foi organizado de acordo com [3] em cinco etapas: (1) definição do estudo de caso; (2) planejamento; (3) operação; (4) análise e interpretação dos dados e; (5) resultados. A Seção 5 descreve os pormenores desse procedimento metodológico.

\section{A ACESSIBILIDADE DE DOCUMENTOS PDF}

O PDF é um tipo de documento digital descendente do Postscript. Ele se destaca pela apresentação fiel do conteúdo e a portabilidade [4]. O conteúdo de um PDF pode integrar elementos variados como textos, imagens, tabelas, gráficos, fórmulas matemáticas, links e formulários, além da estrutura, que determina o encadeamento lógico de todos esses elementos.

Um PDF pode ser originado de processos de escaneamento, conversão, ou pela criação com ferramentas específicas de edição de PDF. No primeiro caso, dos documentos escaneados, o PDF resultante pode ser uma imagem (não acessível) ou um texto com processamento de caracteres ou OCR (Optical Character Recognition). Nesse caso, o PDF tem um pouca de acessibilidade, principalmente por causa da falta de semântica da estrutura do texto [4]. A informação semântica pode conter uma variedade de metadados descritivos, como por exemplo, texto alternativo para imagens [5].

O processo de conversão de arquivos em PDF utiliza softwares de produtividade como os editores de texto, planilhas eletrônicas, editores de apresentação multimídia. Do mesmo modo, é possível converter páginas de Internet em arquivos PDF. Nesses casos, somente a ordem de leitura correta é satisfatória [4], contudo, não é possível afirmar que todos os softwares de produtividade garantem essa característica de acessibilidade dos PDF.

Por fim, as ferramentas específicas de edição de PDF e de sistema de preparação de documentos (LaTeX) que geram os chamados PDF marcados (Tagged PDF). Neles, o texto é ordenado e existem marcas (tags) estruturais para definir cabeçalhos, tabelas, listas, imagens e outros elementos. Com essa codificação, uma tecnologia assistiva (como um leitor de telas) pode apresentar um resumo do documento, facilitar a navegação, fornecer informações estruturais do conteúdo, entre outras coisas [4, 6].

A acessibilidade de PDF foi formalizada pela ISO 14289 [5], que estabeleceu o PDF/UA (PDF Universal Accessibility) [5], cujos requisitos de acessibilidade são organizados em três categorias: (1) requisitos de formato de arquivo; (2) requisitos para programas PDF compatíveis e; (3) Requisitos para tecnologia assistiva compatível [6].

No primeiro caso, dos requisitos de formato de arquivo, o conteúdo do PDF é classificado como significativo e artefatos. Os artefatos são elementos decorativos da página que precisam apenas ser marcados como tais. Já o conteúdo significativo deve ser marcado e integrado na árvore de estrutura de todas as marcas em um documento. Nesse caso, a estrutura em árvore criada pelas tags do documento deve refletir a ordem de leitura lógica do documento [6].

Além disso, outros requisitos, tais como as diretrizes do WCAG (Web Content Accessibility Guidelines), que é a principal recomendação de acessibilidade Web, são destacadas como requisitos de formato de arquivo, quais sejam:
- As informações não podem ser transmitidas apenas por meios visuais (por exemplo, contraste, cor ou posição na página) [6]. Análogo à diretriz do WCAG 2.1, 1.4 Distinguível: Facilite para os usuários ver e ouvir o conteúdo, incluindo a separação do primeiro plano do segundo plano [7].

- Não é permitido conteúdo oscilante ou intermitente, seja como efeitos controlados por favaScript ou como parte de quaisquer vídeos incorporados ao PDF [6]. Referente à diretriz do WGAC 2.1, 2.3 Apreensões e Reações Físicas: Não crie conteúdo que possa causar convulsões ou reações físicas [7].

- Um título de documento deve ser fornecido. O documento deve ser configurado de forma que o título (ao invés do nome do arquivo) apareça no título da janela [6]. Esse requisito está contido nos critérios de sucesso do WCAG 2.1 da diretriz 2.4 Navegável: Forneça maneiras de ajudar os usuários a navegar, encontrar conteúdo e determinar onde eles estão [7].

- O idioma de todo o conteúdo deve ser observado e as alterações de idioma devem ser explicitamente marcadas como tal [6]. No WCAG 2.1, esse requisito faz parte da diretriz 3.1 Legível: Torne o conteúdo do texto legível e compreensível [7].

- Quaisquer elementos pictóricos - sejam objetos de imagem ou outros objetos não textuais, como objetos vetoriais ou grupos de objetos - devem ter um texto alternativo correspondente [6]. No WCAG 2.1, essa é a diretriz 1.1 Alternativas de Texto: Forneça alternativas de texto para qualquer conteúdo que não seja de texto para que ele possa ser alterado para outras formas de que as pessoas precisam como letras grandes, braille, fala, símbolos ou linguagem mais simples [7].

Os requisitos para programas PDF compatíveis (2), dizem respeito às premissas para que a leitura de PDF seja acessível. A leitura de PDF utiliza programas específicos de PDF e navegadores Web. Alguns programas de PDF possuem nativamente um leitor de conteúdo. Para os outros programas sem essa funcionalidade e para os PDF lidos a partir de navegadores Web, tecnologias assistivas como leitores de tela são utilizados. Logo, as ferramentas de leitura de PDF devem estar em conformidade com o PDF/UA para que o conteúdo e outras informações do documento sejam interpretadas pelas tecnologias assistivas. Nesse caso os programas PDF devem [6]:

- Ser capaz de ler e transmitir todas as informações armazenadas nas tags e na árvore de estrutura do documento ou, de outra forma, tornar acessível todo o conteúdo do documento.

- Disponibilizar todo o conteúdo para a tecnologia assistiva, incluindo informações estruturais.

- Permitir que a tecnologia assistiva reconheça o idioma usado e quaisquer alterações no idioma dentro do documento.

- Permitir a navegação no documento por número de página, pela árvore de estrutura ou por favoritos.

Além disso, os programas PDF não devem limitar a funcionalidade da tecnologia assistiva e nem reproduzir conteúdo de mídia automaticamente [6]. 
Quanto aos requisitos para tecnologia assistiva compatível (3), é importante que elas: sejam capazes de reconhecer todos os elementos estruturais, atributos e valores-chave usados na especificação e gerá-los para o usuário de um documento PDF; permitam que o usuário navegue pelo documento por número de página, pela árvore de estrutura ou usando marcadores e que; permitam que o usuário defina e altere facilmente a ampliação de um documento PDF a qualquer momento [6].

\section{TRABALHOS RELACIONADOS}

Conforme mencionado na Seção 2, uma revisão de literatura foi realizada para identificar trabalhos correlatos sobre avaliação de acessibilidade de PDF baseando-se na metodologia de Okoli e Schabram [2]. Como resultado, quatro publicações foram consideradas mais adequadas e são apresentadas a seguir.

A primeira delas foi uma pesquisa realizada por Nganji [8], publicada no artigo "The Portable Document Format (PDF) accessibility practice of four journal publishers". Este estudo avaliou a acessibilidade de 200 artigos em PDF publicados em periódicos de pesquisas relacionadas a pessoas com algum comprometimento. As avaliações utilizaram ferramentas automatizadas e testes manuais com especialistas. Os resultados da avaliação mostraram que a maioria dos artigos tinham problemas de acessibilidade. Dos problemas apontados, cerca de $97 \%$ eram relacionados à falta de texto alternativo para imagens.

Em 2018, Nganji publicou outros dois artigos sobre a temática: "Supporting the information journey of students with disabilities through accessible learning materials" [9] e "An assessment of the accessibility of PDF versions of selected journal articles published in a WCAG 2.0 era (2014-2018)" [10]. Em [9], Nganji utilizou uma amostra de 400 artigos publicados entre 2009-2018 de quatro periódicos. A avaliação considerou o WCAG 2.0 e o uso de ferramenta de avaliação de acessibilidade e leitor de telas. Os resultados apontaram que as versões em PDF dos artigos de periódicos selecionados não eram acessíveis para usuários com leitor de telas, contudo, o autor ponderou que os PDF poderiam ser melhorados com a adoção de práticas acessíveis e inclusivas. Ele ainda indicou a utilização de formatos alternativos como HTML (Hypertext Markup Language) e EPUB (Electronic Publication).

Já em [10], Nganji avaliou 50 artigos indexados no Web of Science entre 2014 e 2018 usando inspeção manual com o Adobe Acrobat Pro XI, PAC 3 (PDF Accessibility Checker 3) e o leitor de telas NVDA (NonVisual Desktop Access) ${ }^{2}$. Os resultados mostraram que apenas $15,5 \%$ dos documentos estavam marcados e $10,5 \%$ tinham texto alternativo para as imagens. Contudo $74,5 \%$ possuíam marcadores para facilitar a navegação e $87 \%$ possuíam títulos significativos nos campos de título. No entanto, de acordo o autor, os textos alternativos das imagens não faziam sentido e os campos de título não eram exibidos quando o documento era aberto, na barra de títulos. Ele constatou que as publicações tinham permissões de acessibilidade habilitadas, o que permitiu a utilização do recurso de leitura em voz alta do Adobe Acrobat Pro XI e do leitor de telas NVDA e que todos os artigos tinham uma versão alternativa em HTML do texto completo no mesmo local do site que as versões em $\mathrm{PDF}$, o que são aspectos positivos. $\mathrm{O}$ autor novamente ponderou que

\footnotetext{
${ }^{2}$ https://sourceforge.net/projects/nvda/
}

o processo de criação dos PDF pode ser melhorado para contemplar a acessibilidade.

Já o artigo "Accessibility of Portable Document Format in Education Repositories" publicado em 2017 por Acosta-Vargas, Luján-Mora e Acosta [11], realizou um estudo de caso de avaliação de acessibilidade de PDF utilizando o PAC 2.02. Os PDF avaliados foram extraídos de repositórios de dez universidades da América Latina. Em cada repositório foram recolhidos aleatoriamente um tutorial e uma tese. Os resultados apontaram que os PDF eram parcialmente acessíveis, ou seja, continham algum erro de acessibilidade, mesmo usando tags, e indicou também documentos sem qualquer marcação. Os autores recomendaram que é necessário que as universidades melhorem o acesso aos documentos PDF, abordando a acessibilidade numa perspectiva de comunicação global, aplicando os critérios de acordo com o WCAG 2.0.

Os trabalhos correlatos apresentados apoiaram a compreensão do estado da arte sobre a avaliação de acessibilidade de PDF. Todos eles apontaram problemas de acessibilidade em PDF em documentos científicos e acadêmicos, o que reforçou a necessidade de verificar o status quo de uma amostra de documentos acadêmicos, geralmente produzidos a partir de softwares de apresentação de slides, conforme apresentado no estudo de caso na próxima Seção.

\section{ESTUDO DE CASO}

De acordo com o que foi apresentado na Metodologia (Seção 2), a organização do estudo de caso baseou-se na metodologia de [3], conforme mostra a Figura 1.

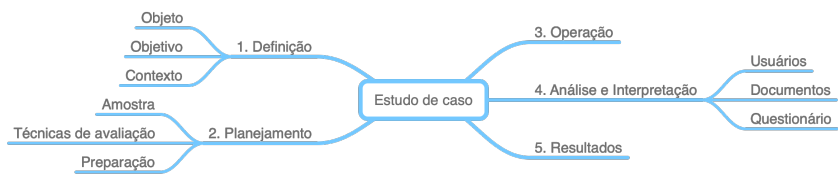

Figure 1: Etapas do estudo de caso

\subsection{Definição}

Nesta etapa determinou-se o objeto, que são documentos PDF; o objetivo que é avaliar a acessibilidade dos documentos PDF e; o contexto que são materiais disponíveis em um AVA de ensino superior.

\subsection{Planejamento}

Nesta etapa realizou-se as seguintes atividades: (a) definição da amostra; (b) seleção da técnica de avaliação de acessibilidade; (c) preparação da avaliação.

5.2.1 Amostra. Na definição da amostra selecionou-se cinco documentos PDF diferentes, referentes a cinco disciplinas obrigatórias de cursos de computação de uma universidade, quais sejam: Sistemas de Apoio à Decisão, Inteligência Artificial, Banco de Dados II, Cálculo Diferencial e Integral III e Algoritmos e Estrutura de Dados I. No caso dessa universidade, os professores divulgam os materiais das disciplinas no AVA Moodle como apoio ao ensino presencial.

Ainda na escolha da amostra, considerou-se a existência de elementos que podem ser eventuais fontes de problemas de acessibilidade. Logo, os documentos PDF coletados na amostra eram 
apresentações de aulas e tinham elementos como tópicos, imagens, gráficos, tabelas, palavras estrangeiras e expressões matemáticas, conforme mostra a tabela 1 .

5.2.2 Seleção das Técnicas de Avaliação. O método proposto compreende uma abordagem híbrida, combinando características e técnicas do teste de usabilidade com a avaliação de acessibilidade.

Os testes de usabilidade objetivam avaliar a qualidade da interação dos usuários com o sistema, o qual nessa pesquisa consiste em um documento PDF. São testes realizados em laboratório, onde é avaliado o desempenho de usuários típicos nas tarefas selecionadas e preparadas para o teste, e que produzem dados para análise como métricas (número de erros, tempo de realização de cada tarefa), $\log s$, vídeos, anotações e questionários [12]. Geralmente adotam a observação e seu registro criterioso como principal fundamento teórico-metodológico, de forma a possibilitar a comunicação e avaliação do grau de severidade dos problemas encontrados. A observação científica do comportamento do usuário (postura corporal, gestos e expressões), sem o viés de interpretações do observador, fornecem indicadores úteis para a descrição dos eventos de interação (sucesso, fracasso, erro) e em que contexto eles ocorreram[13] Segundo Preece (2005), observações podem ser (a) exploratória (sem objetivos predefinidos), (b) sistemática (focada em objetivos) e (c) protocolada (pautada em indicadores preestabelecidos) [12]. Nesse estudo, as observações foram sistemáticas, focadas na avaliação da acessibilidade dos elementos considerados críticos nos documentos PDF.

Para guiar o teste de usabilidade adotou-se o framework DECIDE, proposto em [12], que consiste nas seguintes etapas: (1) Determine, que estabelece as metas e os objetivos da avaliação. (2) Explore, que determina as questões a serem respondidas pela avaliação. (3)Choose, que escolhe os métodos e as técnicas que responderão às questões da avaliação. (4) Identify, que busca identificar questões práticas a serem abordadas pela avaliação. (5) Decide, que decide como lidar com as questões éticas envolvidas. Por fim, (6) Evaluate, para avaliar, interpretar e apresentar os dados. A Figura 2 mostra o framework DECIDE para o teste de usabilidade aqui apresentado.

O framework DECIDE incorporou técnicas da avaliação de acessibilidade Web como referencial para a avaliação de acessibilidade de PDF. A avaliação de acessibilidade Web verifica o quão bem um site pode ser utilizado por pessoas com algum comprometimento. Esta avaliação pode valer-se de ferramentas automatizadas, do julgamento de especialistas e de testes com usuários [14]. Esse último possibilita a observação das estratégias de interação construídas pelos diferentes usuários na realização de tarefas típicas e com o uso de tecnologias assistivas (como os leitores de tela), bem como a identificação das dificuldades que eles enfrentam [15] apud [16] Os tipos de avaliação de acessibilidade podem ser combinados ou utilizados isoladamente. Nessa pesquisa, optou-se pela avaliação com usuários.

Abou-Zahra [14] apresenta alguns paradigmas de avaliação de acessibilidade com usuários, tais como: medidas de desempenho, registro das ações do usuário, entrevista, questionário, observação, e a técnica "pensar em voz alta". Desses, essa pesquisa combinou os três últimos paradigmas citados: questionário, observação e a técnica "pensar em voz alta", assim como especificado na etapa Choose do framework DECIDE.

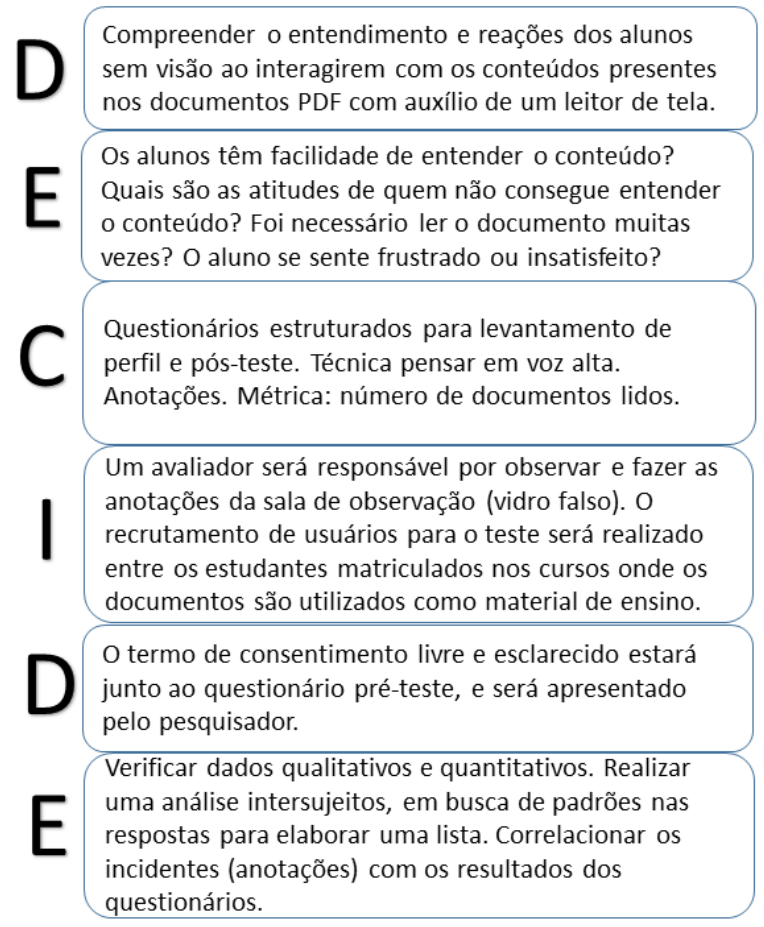

Figure 2: Teste de usabilidade de documentos PDF

Os questionários captam a percepção dos usuários com relação ao sistema ou a interface. Eles podem ser realizados com questões abertas ou fechadas. Já a técnica de observação verifica diretamente o comportamento do usuário notando aspectos sobre como uma interface ajuda ou impede a sua interação e revela problemas para completar tarefas específicas. Por fim, na técnica "pensar em voz alta" os usuários são observados usando a interface enquanto verbalizam suas ideias, sentimentos e opiniões [14].

5.2.3 Preparação da Avaliação. Nesta fase definiu-se: (1) a quantidade de usuários: sete e; (2) o tipo de comprometimento usuário: cegueira.

Os usuários foram divididos em dois grupos: grupo piloto e grupo participante, em que no primeiro participaram duas pessoas em uma análise prévia, para verificar se a dinâmica de avaliação elaborada estava em conformidade com os objetivos da pesquisa. Já na avaliação com o grupo participante, foram avaliadas as interações de cinco pessoas, totalizando sete usuários envolvidos no estudo.

Para as avaliações de acessibilidade com usuários, adotou-se a mesma abordagem de [17] em que os usuários vendados participaram dos testes preliminares para verificar a viabilidade da proposta. No entanto, no caso desta pesquisa, esta estratégia foi necessária não somente nos testes preliminares ( $1^{\circ}$ grupo), mas nos dois grupos, visto que não havia usuários com cegueira para participar da investigação. 
XII Computer on the Beach

\begin{tabular}{lccccc}
\hline Disciplina & Tópico & Imagem / gráfico & Tabela & Palavra estrangeira & Expressão matemática \\
\hline Sistemas de Apoio à Decisão & Sim & Sim & Sim & Não & Não \\
Inteligência Artificial & Sim & Sim & Sim & Não & Não \\
Banco de Dados II & Sim & Não & Não & Sim & Sim \\
Cálculo Diferencial e Integral III & Sim & Sim & Não & Sim & Sim \\
Algoritmos e Estruturas de Dados I & Sim & Não & Não & Sim & Não \\
\hline
\end{tabular}

Table 1: Características (elementos) dos documentos PDF da amostra

Na sequência, determinou-se a tecnologia assistiva a ser utilizada pelo usuário, a saber, leitor de telas NVDA e o agente de usuário, navegador web Chrome ${ }^{3}$.

Os questionários pré-teste e pós-teste foram elaborados, assim como um conjunto de 14 anotações (ver tabela 2) para facilitar a observação das ações e reações dos usuários durante a execução da avaliação. Essas anotações buscaram refletir os comportamentos e sentimentos durante a interação dos usuários. As anotações A3 até A8 dizem respeito aos elementos do PDF e as demais sobre as reações dos usuários na interação.

\subsection{Operação}

Uma vez que o projeto foi aprovado pelo Comitê de Ética em Pesquisa da Universidade, iniciou-se a terceira etapa do estudo de caso. Esta fase ocorreu em um laboratório da universidade devidamente preparado para a avaliação.

Cada usuário realizou a avaliação individualmente com o acompanhamento de uma avaliadora. Antes de porém, eles assinaram o Termo de Consentimento Livre e Esclarecido e preencheram o questionário pré-teste, conforme a fase Decide apresentado na Figura 2.

Os usuários interagiram com o PDF de olhos vendados. Usando a técnica "pensar em voz alta", eles foram orientados a se expressarem durante a avaliação, enquanto isso as observações das interações foram registradas nas anotações apresentadas na Tabela 2. O avaliador também registrou a frequência de ocorrência das anotações para cada usuário em cada documento.

Após a sessão de avaliação de acessibilidade, os usuários responderam um questionário pós-teste com o objetivo de avaliar seu entendimento quanto aos conteúdos abordados nos documentos PDF.

\subsection{Análise e interpretação dos dados}

Em face dos dados coletados nos questionário pré-teste, na observação do avaliador e no questionário pós-teste de cada usuário, iniciou-se a análise e interpretação dos dados.

Com relação às anotações do avaliador durante a observação das interações dos usuários, como pode ser observado na figura 3, todas elas ocorreram durante a avaliação, contudo, a anotação A13 (O usuário não entende alguma informação e tenta ouvi-la novamente) foi a que mais ocorreu, seguida da $\mathrm{A} 10$ (O usuário não entende alguma informação e não se conforma com isso) e A3 (O usuário não consegue identificar um tópico). As próximas subseções abordam a análise e interpretação dos usuários, coletados

\footnotetext{
${ }^{3}$ https://www.google.pt/intl/pt-PT/chrome/
}

no questionário pré-teste, dos documentos e do questionário pósteste, respectivamente.

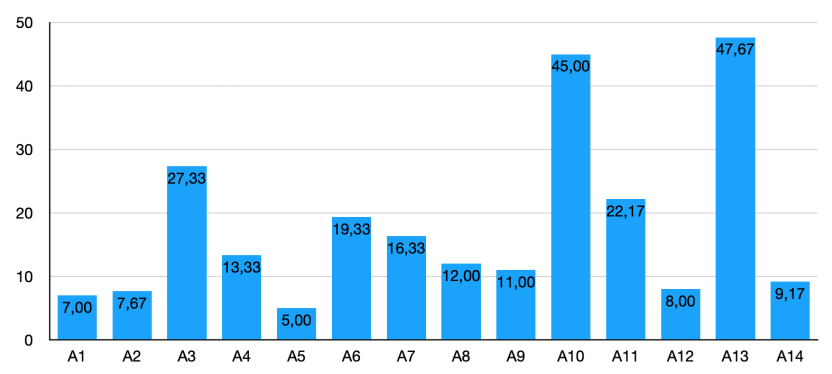

Figure 3: Média de ocorrência de anotações

5.4.1 Análise e interpretação dos dados dos usuários. Com relação aos dados do perfil dos usuários coletados no questionário pré-teste, $43 \%$ são do sexo feminino, $43 \%$ masculino e $14 \%$ não declararam. Quanto à faixa etária, a maioria tinha entre 18 e 25 anos (87\%). Sobre a escolaridade, $86 \%$ estavam cursando o ensino superior e $14 \%$ tinham o ensino médio completo.

Sobre a experiência no uso do computador, a maioria $(71,4 \%)$ afirmou ter mais de 15 anos de experiência no uso do computador. Quanto ao acesso diário ao computador, $86 \%$ declarou utilizar o computador por mais de 6 horas por dia. Além disso, todos afirmaram realizar mais frequentemente leitura de material digital na Internet do que em material físico (papel). Nesse caso, é unânime o uso do PDF.

Conforme mencionado, não houveram usuários cegos na avaliação. Contudo, $86 \%$ dos participantes declararam ter problemas de visão como miopia, hipermetropia ou astigmatismo. Os demais mencionaram não ter problemas de visão. Mesmo assim, $57 \%$ afirmaram já terem utilizado leitores de tela. Entre os leitores de telas citados pelos usuários estão as extensões dos navegadores Chrome e Microsoft Edge, e os leitores Jaws e NVDA.

Dos problemas que os usuários tiveram, os que mais ocorreram foram as anotações 11 (o usuário não entende alguma informação e tenta ouvi-la novamente), 10 (o usuário não entende alguma informação e não se conforma com isso) e 3 (usuário não consegue identificar um tópico). O documento de leitura mais fácil foi o 1 . Foi observado que esse documento não tinha palavras estrangeiras, nem expressões matemáticas.

A média de frequência de anotações por usuário foi de 3,5. Como mostra o gráfico na figura 4, o usuário 7 teve a maior média de ocorrências, o que indica que ele teve mais dificuldades durante 


\begin{tabular}{ll}
\hline ID & Anotação \\
\hline A1 & O usuário sabe a operação que deseja realizar, mas não encontra de imediato \\
A2 & O usuário não sabe o que fazer e busca o próximo passo \\
A3 & O usuário não consegue identificar um tópico \\
A4 & O usuário não consegue identificar uma imagem \\
A5 & O usuário não consegue identificar um gráfico \\
A6 & O usuário não consegue identificar uma palavra estrangeira \\
A7 & O usuário não consegue identificar uma expressão matemática \\
A8 & O usuário não consegue identificar uma tabela \\
A9 & O usuário realizou uma ação indesejada \\
A10 & O usuário não entende alguma informação e não se conforma com isso \\
A11 & O usuário fica impaciente e começa a mexer no mouse ou no teclado \\
A12 & O usuário não consegue realizar uma tarefa e pede auxílio \\
A13 & O usuário não entende alguma informação e tenta ouvi-la novamente \\
A14 & O usuário não consegue terminar a tarefa e desiste \\
\hline
\end{tabular}

\section{Table 2: Anotações das interações}

a interação com o PDF. Do contrário, o usuário 4 teve menos anotações observadas, logo menos dificuldades durante a interação. Contudo, ao terminar a interação com o segundo documento, o usuário 4 desistiu da avaliação, justificando ter se sentido esgotado ao tentar entender os conteúdos. Esse mesmo argumento foi utilizado pelo usuário 2, que desistiu ao terminar a interação com o documento 3 .

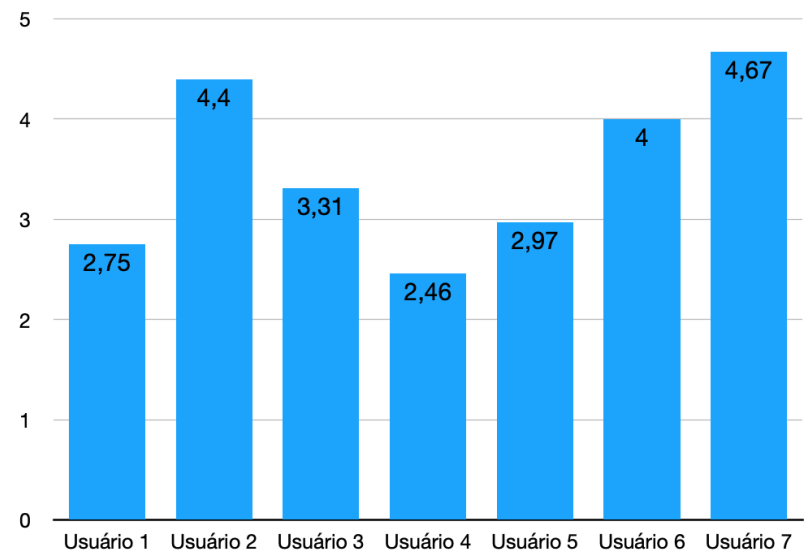

Figure 4: Média de ocorrência de anotações por usuário

5.4.2 Análise e interpretação dos dados dos documentos. Todos os documentos PDF da amostra tinham tópicos, $80 \%$ tinham palavras estrangeiras, $60 \%$ imagens e/ou gráficos e $40 \%$ tinham tabelas e fórmulas. O grau de dificuldade identificado nas interações dos usuários com eles foi praticamente o mesmo, como mostra o gráfico com a média de anotações por documento, na figura 5. Como pode ser observado nesta figura, o Documento 1 teve uma ligeira alta de anotações, isto porque, de acordo com a observação do avaliador, frente ao primeiro contato com a leitura de PDF por meio de leitor de telas, os usuários tiveram dificuldades em iniciar a interação sem o auxílio do avaliador, mesmo aqueles que disseram ter experiência com leitores de tela. Além disso, os participantes tiveram dificuldades em identificar os tópicos, imagens e tabelas presentes no documento. Nesse PDF ocorreu com mais frequência a anotação A10 (o usuário não entende alguma informação e não se conforma com isso).

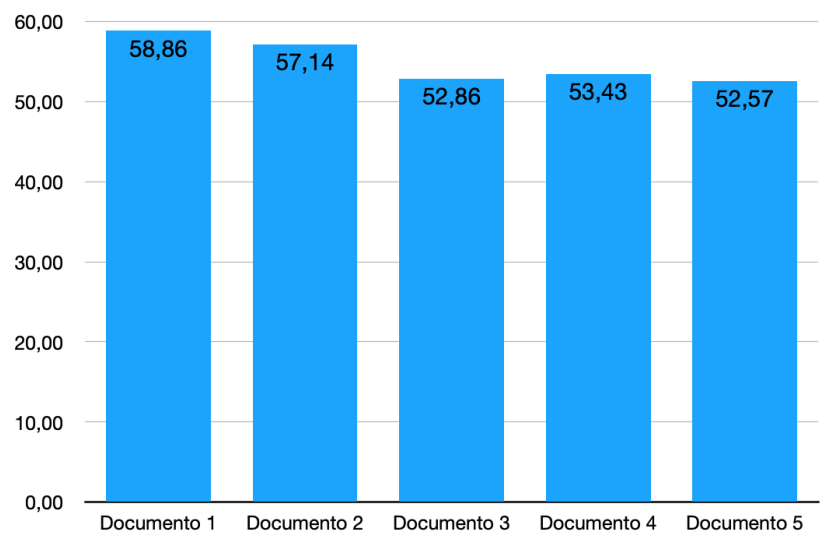

Figure 5: Média de ocorrência de anotações por documento

Na interação com o Documento 2, além da anotação A10, a A13 também ocorreu com muita frequência (o usuário não entende alguma informação e tenta ouvi-la novamente). Contudo, os participantes relataram que a leitura de tópicos foi mais tranquila em relação ao documento anterior, pois eles já estavam familiarizados com a terminologia adotada pelo leitor de tela para aquele elemento.

Na interação com o documento 3, um dos participantes (o usuário 4) desistiu de prosseguir com a avaliação e justificou se "sentir esgotado" ao tentar identificar os conteúdos. Assim como no documento 2, no documento 3 as anotações que mais ocorreram foram a A13 e A10, seguida pela A11 (o usuário fica impaciente e começa a mexer no teclado ou mouse). De acordo com uma participante, a sensação era de não saber ler: "À medida que vou tentando entender o que o 
conteúdo está querendo me passar, fico nervosa e cansada. É como se eu não soubesse realmente ler". Ao final da interação com esse documento, o usuário 2 desistiu de continuar na avaliação.

$\mathrm{Na}$ análise da interação dos usuários com o Documento 4, os participantes que permaneceram no teste relataram que nenhuma das expressões matemáticas contidas no documento foram entendidas (A7). Além disso, eles se sentiram irritados pois, nas palavras deles, "as letras se repetiam". Entretanto, dois usuários declararam que a leitura deste documento foi a mais fluida e compreensível, ainda que a maioria não tenha identificado corretamente todos os tópicos (A3). Na fala de um dos participantes: "Não dá para saber se já finalizou um assunto ou se está no mesmo".

Por fim, na avaliação com o Documento 5, somente dois participantes leram o documento até o final, uma vez que os demais decidiram não prosseguir com a avaliação, justificando a desistência pelo desgaste e sensação de que os conteúdos do documento se repetiam a todo momento. Observou-se uma alta frequencia de A6 (problemas coom leitura de palavra estrangeira) e A11 (usuário fica impaciente e mexe no mouse e teclado).

5.4.3 Análise e interpretação do questionário pós-teste. Ao finalizar a avaliação, cada usuário (mesmo os que desistiram da avaliação) preencheu o questionário pós-teste com questões de opinião utilizando a escala de Likert. Essa avaliação foi importante para corroborar as observações do avaliador.

Como pode ser observado na tabela 3 , apenas os tópicos foram parcialmente compreendidos por $14,3 \%$ usuários. Para todos os seis elementos analisados, a maioria dos usuários responderam que não os compreenderam.

Outras duas questões do questionário pós-teste apontaram que $100 \%$ dos usuários precisariam da ajuda de outras pessoas para compreender melhor os conteúdos dos documentos. Do mesmo modo, eles não se sentiram confiantes durante a interação com os documentos PDF.

\subsection{Resultados do estudo de caso}

Os dados levantados durante a observação do avaliador utilizando a técnica "pensar em voz alta" combinada com um conjunto de anotações das interações apontaram que recorrentemente os usuários não entenderam alguma informação no documento PDF e tentaram ouvi-la novamente com o leitor de telas. Constatou-se também que o usuário sentia-se inconformado quando não conseguia compreender uma informação. Isso foi corroborado pelo questionário pós-teste, pois os usuários relataram precisar de ajuda para compreender os conteúdos por não se sentirem confiantes durante a interação.

Com relação aos elementos, devido à característica dos documentos, apresentações de aulas, todos eles tinham conteúdos na forma de tópicos e os usuários algumas vezes não conseguiam compreende-los. Apesar disso, tópico foi o único elemento que teve respostas positivas quanto ao seu entendimento na avaliação pós-teste.

O estudo de caso também revelou que mesmo os usuários sendo experientes no uso do computador, eles tiveram dificuldades de compreender os conteúdos dos documentos PDF com os olhos vendados e utilizando leitor de telas.
A desistência de dois usuários foi um fator relevante para a avaliação. Esses usuários sentiram-se "esgotados" ao tentarem entender o conteúdo. Isso mostra o quanto a leitura de um documento PDF inacessível pode desmotivar uma pessoa com comprometimento visual de prosseguir com os estudos, uma vez que o escopo deste artigo foi o ambiente acadêmico. Ainda, considerando que os usuários têm boa experiência com computador e o utilizam por muitas horas durante o dia, entende-se que a experiência com computador não foi um fator determinante nesse resultado.

Outro resultado importante, diz respeito às expressões matemáticas. Apesar delas estarem presentes em dois dos cinco documentos da amostra, elas são um elemento importante no contexto do artigo por se tratar de um instituto de ciências exatas, logo a matemática está presente em muitas disciplinas ofertadas.

Por fim, assim como as expressões matemáticas e os tópicos, as tabelas, imagens e gráficos são recursos amplamente utilizados em apresentações de aula por ajudarem na representação do conhecimento e no entendimento dos alunos, contudo, esse último não foi possível para alunos que realizaram a avaliação simulando cegueira.

\section{CONSIDERAÇÕES FINAIS}

Documentos PDF são amplamente utilizados no meio acadêmico e compartilhados na Internet devido ao fato de manterem suas características originais independente do ambiente de visualização. Nesse sentido, é importante que, assim como uma página Web construída em HTML deve ser acessível para todas as pessoas, os documentos PDF também devem cumprir requisitos de acessibilidade.

$\mathrm{Na}$ amostra utilizada no estudo de caso, concluiu-se que os documentos PDF disponibilizados no AVA Moodle como material de apoio ao ensino presencial são inacessíveis para os alunos nas condições apresentadas na avaliação (com os olhos vendados), o que pode indicar que os documentos também são inacessíveis para pessoas com cegueira. Embora os usuários estivessem vendados, os resultados obtidos com pessoas normovisuais podem ser diferentes se realizados com pessoas com deficiência visual. Isso porque, os primeiros, geralmente, apresentam dificuldades em utilizar leitores de tela, portanto, os resultados obtidos podem não ser consequência apenas da falta de acessibilidade, mas também da dificuldde de uso desse tipo de leitor.

As barreiras de acessibilidade encontradas dizem respeito aos conteúdos expressos em tabelas, gráficos, imagens, expressões matemáticas e às palavras estrangeiras. Esses elementos, particularmente, são frequentes em cursos da área de exatas. Nesse sentido, é necessário propor ações para sensibilizar os professores para os problemas de acessibilidade na leitura de documentos PDF, além de estudos sobre os softwares utilizados para produzir material de ensino e como eles impactam a acessibilidade dos documentos PDF.

A metodologia criada para a avaliação de acessibilidade dos documentos PDF utilizando uma relação de anotações de problemas e a frequência que eles aconteceram foi elaborada a partir de técnicas utilizadas na avaliação de usabilidade de interfaces com usuários. Ela mostrou-se simples e adequada ao estudo, contudo, futuras avaliações com usuários utilizarão um checklist baseado nas recomendações do WCAG e ferramentas de avaliação de acessibilidade de PDF. Do mesmo modo, os documentos serão avaliados 
XII Computer on the Beach

\begin{tabular}{lllll}
\hline Questão & $\begin{array}{l}\text { Discordo } \\
\text { totalmente }\end{array}$ & $\begin{array}{l}\text { Discordo parcial- } \\
\text { mente }\end{array}$ & $\begin{array}{l}\text { Indiferente } \\
\text { cialmente }\end{array}$ & $\begin{array}{l}\text { Concordo par- } \\
\text { concordo to- } \\
\text { talmente }\end{array}$ \\
\hline Compreendi os tópicos & $42,9 \%$ & & $42,9 \%$ & $14,3 \%$ \\
Compreendi as imagens & $100 \%$ & & \\
Compreendi os gráficos & $100 \%$ & & \\
Compreendi as tabelas & $87 \%$ & $14,3 \%$ & & \\
Compreendi as expressões matemáticas & $100 \%$ & & & \\
Compreendi as palavras estrangeiras & $42,9 \%$ & $28,6 \%$ & $28,6 \%$ & \\
\hline
\end{tabular}

Table 3: Dados do questionário pós-teste

em ordem aleatória para investigar o impacto dos elementos do documento nas desistências dos usuários.

Além disso, atualmente, estão sendo planejados novos testes para estender e cobrir um número maior de usuários e perfis, a saber: usuários vendados, usuários com baixa visão, e usuários totalmente cegos. Ademais, análises sobre os efeitos da aprendizagem no desempenho do resultado dos participantes estão sendo consideradas. Instrumentos para identificar os possíveis efeitos de sobrecarga de informação e fadiga foram mapeados para utilização em estudos futuros.

Por fim, o trabalho possibilitou conhecer os problemas de acessibilidade dos documentos PDF utilizados como apoio ao ensino presencial em cursos da área de tecnologia, problemas esses que podem se tornar barreiras para o acesso de todos ao conhecimento. Outrossim, o estudo ressalta a importância da acessibilidade não somente das páginas construídas em HTML, mas também em PDF, muito utilizado no meio acadêmico.

\section{REFERENCES}

[1] W3C-Brasil. Cartilha de acessibilidade na web. https://www.w3c.br/pub/ Materiais/PublicacoesW3C/cartilha-w3cbr-acessibilidade-web-fasciculoI.html\#capitulo2. Accessed: 2020-07-01.

[2] Chitu Okoli and Kira Schabram. A guide to conducting a systematic literature review of information systems research. 2010.

[3] Claes Wohlin, Per Runeson, Martin Höst, Magnus C Ohlsson, Björn Regnell, and Anders Wesslén. Experimentation in software engineering. Springer Science \& Business Media, 2012.

[4] Mireia Ribera Turró. Are pdf documents accessible? Information Technology and Libraries, 27(3):25-43, 2008.

[5] Iso 14289-1:2014(en) - document management applications - electronic document file format enhancement for accessibility - part 1: Use of iso 32000-1 (pdf/ua-1) 2014

[6] O Drümmer and B Chang. Pdf/ua in a nutshell. Accessible documents with PDF, 2012.

[7] W3C. Web content acessibility guidelines 2.1. < https://www.w3.org/TR/ WCAG21/, 2018. Accessed: 2020-12-08.

[8] Julius T. Nganji. An assessment of the accessibility of pdf versions of selected journal articles published in a wcag 2.0 era (2014-2018). Learned Publishing, 31 (4):391-401, 2015. doi: 10.1002/leap.1197. URL https://onlinelibrary.wiley.com/ doi/abs/10.1002/leap.1197.

[9] Julius T Nganji. Supporting the information journey of students with disabilities through accessible learning materials. Information and Learning Science, 2018.

[10] Julius T Nganji. An assessment of the accessibility of pdf versions of selected journal articles published in a wcag 2.0 era (2014-2018). Learned Publishing, 31 (4):391-401, 2018 .

[11] Patricia Acosta-Vargas, Sergio Luján-Mora, and Tania Acosta. Accessibility of portable document format in education repositories. In Proceedings of the 2017 9th International Conference on Education Technology and Computers, pages 239-242, 2017.

[12] J. Preece, Y. Rogers, and H. Sharp. Design de Interação: além da interação homem computador. Bookman, 2005.

[13] M. F. Danna and M. A. Matos. Ensinando Observação: Uma Introdução. São Paulo: Edicon, 1999.
[14] Shadi Abou-Zahra. Web accessibility evaluation. In Web accessibility, pages 79-106. Springer, 2008.

[15] Amanda Meincke Melo, M Cecília C Baranauskas, and Fabiana Fator Gouvêa Bonilha. Avaliação de acessibilidade na web com a participação do usuário: um estudo de caso. Simpósio Sobre Fatores Humanos Em Sistemas Computacionais, 6: 165-168, 2004.

[16] Lucinéia Souza Maia. Um processo para o desenvolvimento de aplicações web acessíveis. 2010 .

[17] A. Awada, Y. B. Issa, C. Ghannam, J. Tekli, and R. Chbeir. Towards digital image accessibility for blind users via vibrating touch screen: A feasibility test protocol. In 2012 Eighth International Conference on Signal Image Technology and Internet Based Systems, pages 547-554, 2012. doi: 10.1109/SITIS.2012.85. 